\title{
Stereotactic arrhythmia radiotherapy: a case study of real-time image-guided noninvasive treatment for ventricular tachycardia
}

\author{
Jing Li ${ }^{1 \#}$, Qingyong Chen ${ }^{2 \#}$, Guangjun $\mathrm{Li}^{1 \#}$, Yan $\mathrm{Li}^{3}$, Yingjie Zhang ${ }^{1}$, Changhu $\mathrm{Li}^{1}$, Long Bai ${ }^{1}$, \\ Renming Zhong ${ }^{1}$, Yinbo $\mathrm{He}^{1}$, Feng $\mathrm{Xu}^{3}$, Qing Yang ${ }^{2}$, Sen Bai ${ }^{1}$ \\ ${ }^{1}$ Department of Radiation Oncology, Cancer Center and State Key Laboratory of Biotherapy, West China Hospital, Sichuan University, Chengdu, \\ China; ${ }^{2}$ Department of Cardiology, West China Hospital, Sichuan University, Chengdu, China; ${ }^{3}$ Lung Cancer Center, West China Hospital, Sichuan \\ University, Chengdu, China \\ "These authors contributed equally to this work.
}

Correspondence to: Sen Bai. Department of Radiation Oncology, Cancer Center and State Key Laboratory of Biotherapy, West China Hospital, Sichuan University, Chengdu, China. Email: baisen@scu.edu.cn; Qing Yang. Department of Cardiology, West China Hospital, Sichuan University, Chengdu, China. Email: yangqing@scu.edu.cn; Feng Xu. Lung Cancer Center, West China Hospital, Sichuan University, Chengdu, China. Email: fengxuster@gmail.com.

Submitted Oct 18, 2021. Accepted for publication Dec 10, 2021.

doi: 10.21037/qims-21-1025

View this article at: https://dx.doi.org/10.21037/qims-21-1025

\section{Introduction}

In clinical practice, ventricular tachycardia (VT) is usually treated with antiarrhythmic drugs and catheter ablation. However, drug therapy has a relatively low rate of overall effectiveness and may cause new arrhythmia. Catheter ablation has an approximately $50 \%$ recurrence rate for VT patients with non-ischemic cardiomyopathy 1 year postoperatively (1). In the recent years, non-invasive stereotactic body radiotherapy (SBRT) has been used to treat arrhythmia, and several studies have shown significant reductions in VT episodes with few serious toxicities after a 6-week blanking period (2). However, the persistence of the treatment effect has varied across studies; thus, a scalable workflow of image-guided radiotherapy techniques must be created to improve the accuracy of the approach (3).

This case report describes the SBRT treatment of a patient with VT who had undergone ineffective radiofrequency ablations. The primary purpose of this case report is to propose a method for accurate movement evaluation and tracking of the arrhythmia target through the registration and fusion of multimodal images.

\section{Case presentation}

A 54-year-old man was admitted to the hospital in 2013 for repeated palpitations and syncope. He was diagnosed with VT and cardiogenic syncope caused by dilated cardiomyopathy. A cardiac resynchronization therapydefibrillator (CRT-D) was implanted to prevent sudden cardiac death. However, recurrent episodes of VT forced him to undergo catheter radiofrequency ablations twice in 2018; both of which failed. The patient's most recent visit for abnormal CRT-D discharge revealed that he had a VT burden of 37 events per day with an anti-tachycardia pacing (ATP) frequency of 30 sequences per month and a shock frequency of three events per month. According to the New York Heart Association classification, he had class IV heart failure with a left ventricular ejection fraction (LVEF) of $32 \%$. The invasive electroanatomic mapping (EAM) showed that the patient had an epicardial VT substrate located at the root of the left anterior descending artery. Considering the high risk of coronary artery damage with epicardial ablation, the attending cardiologist performed a third endocardial catheter ablation, which was also 
Table 1 Timeline

\begin{tabular}{ll}
\hline Index date & Event \\
\hline 2013 & Diagnosed as VT and implanted CRT-D \\
2018 & $1^{\text {st }}$ catheter ablation failed \\
2018 & $2^{\text {nd }}$ catheter ablation failed \\
2019 & Replacement of CRT-D battery \\
2021 & $3^{\text {rd }}$ catheter ablation failed \\
2021 & Received stereotactic body radiotherapy \\
After 6-week blanking period & Significant improvement of VT \\
After 6-month post-ablation period & No recurrences of VT and complications \\
\hline VT, ventricular tachyoardia; CRT-D, cardiac resynchronization therapy-defibrillator.
\end{tabular}

VT, ventricular tachycardia; CRT-D, cardiac resynchronization therapy-defibrillator.

unsuccessful. As a result, the patient was referred for SBRT. All procedures performed in this study were in accordance with the ethical standards of the Ethics Committee of West China Hospital of Sichuan University and with the Helsinki Declaration (as revised in 2013). Written informed consent was obtained from the patient for publication of this case report and accompanying images. A copy of the written consent is available for review by the editorial office of this journal. The timeline of his clinical course is summarized in Table 1.

During simulation and treatment, the patient was immobilized in a vacuum bag in the supine position with his arms raised above his head. A large-aperture computed tomography (CT) simulator (Revolution CT, GE Healthcare, Boston, MA, USA) with 128 rows was used for patient simulation. Two scan protocols were applied: (I) free-breathing respiratory four-dimensional CT (r4DCT) images of single respiratory cycles were obtained and reconstructed into 10 phases (from 0 to $90 \%$ ) at equal time intervals with the average intensity projection of the 10 phases calculated as the planning CT (pCT) with a thickness of $2.5 \mathrm{~mm}$; (II) exhale breath-hold coronary CT angiography (CCTA) was implemented and reconstructed at a thickness of $2.5 \mathrm{~mm}$ to effectively reduce the artifacts caused by heartbeats and accurately locate the gross target volume (GTV) of the arrhythmia. The two series of images were imported into the commercial MIM Maestro software (Version 6.8, MIM Software Inc., Cleveland, OH, USA). The cardiologists, who were specialty trained in SBRT, referred to previous EAM results and located the VT origin around the base of the anterior wall of the left ventricle, between segments 1 and 2, based on the 17-segment model of the American Society of Echocardiography (4); subsequently, we delineated the corresponding contour in the CCTA images as shown in Figure 1. To evaluate respiratory motion using $\mathrm{r} 4 \mathrm{DCT}$ with heart motion artifacts, the physicists attempted to register the CCTA with $10 \mathrm{r}$ 4DCT phases. The automatic rigid registration results shown in Figure 2 demonstrate a high degree of overlap between the CCTA and r4DCT-50\% (the end-expiratory phase) images, which rendered manual adjustment almost unnecessary by referring to structures such as the sternum, ribs, diaphragm, and heart. Thus, the GTV was transferred from the CCTA to the r4DCT-50\% images.

Using a deformable image registration algorithm in MIM, the GTV of the other r4DCT phases was generated from the r4DCT-50\% results. After review and modification by the cardiologist, all 10 GTVs were transferred to the pCT and merged to create the internal target volume (ITV), as shown in Figure 3. Taking setup and machine errors into consideration, the ITV was expanded with a margin of $3 \mathrm{~mm}$ to generate a planning target volume (PTV) of $74.7 \mathrm{~mL}$ (2); the position of the PTV relative to the heart is shown in Figure 4. In addition, the organs at risk (OARs) -including the coronary artery, heart (excluding PTV), esophagus, bronchus, lungs, spinal cord, stomach, and liver-were delineated on pCT by the oncologists. The targets and OARs were then imported into the Eclipse treatment planning system (Version 13.5, Varian Medical System Inc., Palo Alto, CA, USA).

The PTV was treated with a prescription radiation dose of 25 Gy delivered in a single fraction by an Edge linear accelerator (Varian Medical Systems) using 6-MV flattening filter beams. The two beams-one from $270^{\circ}$ 

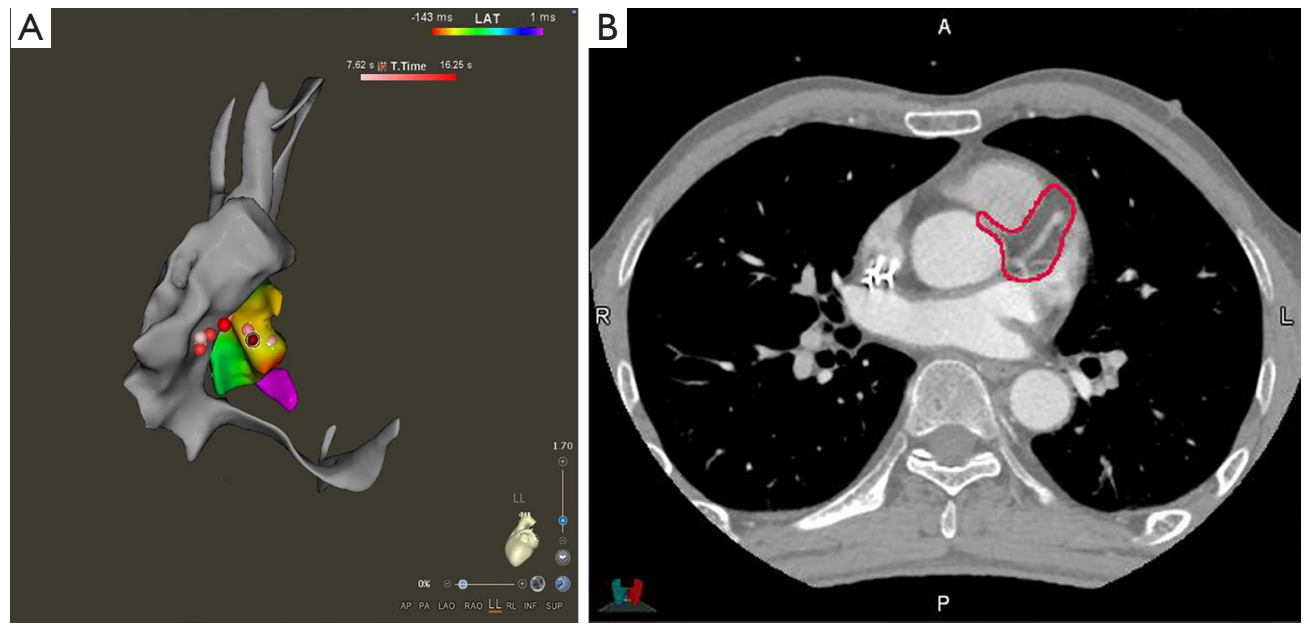

Figure 1 GTV delineated based on EAM result (A) and CCTA images (B). GTV, gross target volume, red line in (B); EAM, electroanatomic mapping; CCTA, coronary CT angiography.

to $150^{\circ}$ clockwise and the other from $150^{\circ}$ to $270^{\circ}$ counterclockwise-were designed using the stereotactic radiosurgery volumetric modulated arc technique. Following reverse optimization and calculation of the dose distribution using the analytical anisotropic algorithm, the plan was evaluated by a senior physicist, oncologist, and cardiologist. The dose distribution, dose volume histogram, and several crucial dose parameters are shown in Figure 4. Using an SRS MapCHECK (Sun Nuclear Corp., Melbourne, FL, USA), further dose verification was performed by comparing the measurement and calculation doses through gamma analysis. The gamma passing rate under an absolute dose criterion of $2 \%$ global dose difference and $2 \mathrm{~mm}$ distance-to-agreement was $99.1 \%$ (3).

After the plan was authorized for treatment application, an initial patient setup was carried out by aligning an isocenter laser with the crosshairs on the vacuum bag. We then obtained a cone beam CT (CBCT) image, registered the CBCT image with the pCT image by referring to the heart edge within the PTV range, spine, and sternum, and corrected the setup error (Figure 5). The corrected CBCT images were used to generate the patient's body surface and a region of interest (ROI) covering the PTV was selected to be the reference for optical surface imaging (OSI). During treatment, the OSI results were interlocked with beam delivery of the linear accelerator to monitor realtime surface movements in the vertical, longitudinal, and lateral directions as well as yaw, roll, and pitch rotations. When the motion amplitude in a certain direction exceeded a threshold of $3 \mathrm{~mm}$, the linear accelerator paused the beam delivery until the motion amplitude returned to a stable state within the threshold, at which point the beam delivery was resumed. As shown in Figure 6, over an 8.75-min course of beam delivery, the patient underwent a single VT episode that produced significant changes in longitudinal movement.

Following SBRT, the patient was closely monitored in the coronary heart disease intensive care unit for 24 hours and was then followed for 6 months. To ensure patient safety, myoglobin, creatine kinase $\mathrm{MB}$, cardiac troponin $\mathrm{T}$, and pro-brain natriuretic peptide levels were assessed on day 2 and week 6 after SBRT with the results indicating that no myocardial damage has occurred. Following a 6-week blanking period, the number of incessant VT episodes decreased from 37 to 2 events per day (as measured by a 24-hour Holter monitor), which represented a 94.59\% reduction in the VT burden, and the LVEF increased from $32 \%$ to $41 \%$. According to the CRT-D pretreatment and post-treatment records, the ATP frequency decreased from 30 sequences per month to none, and the number of shocks decreased from three per month to none, corresponding to $100 \%$ reduction for each event. The patient did not develop any radiotherapy complications during the 6 months of follow-up.

\section{Discussion}

SBRT shows marked potential for use in the noninvasive treatment of patients with VT who have undergone repeated, unsuccessful radiofrequency ablations. 

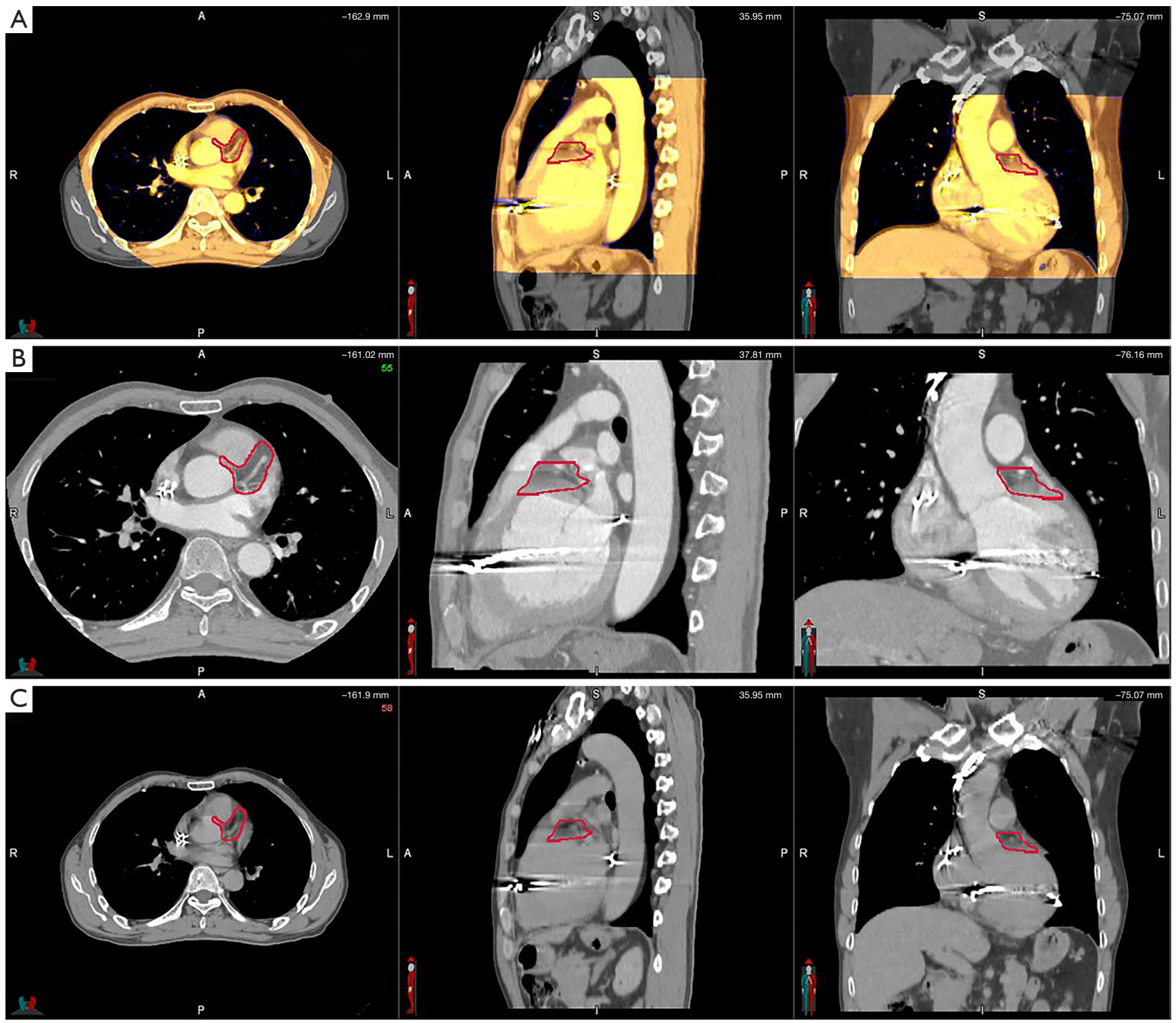

Figure 2 Fusion series (A) of CCTA (B) and r4DCT-50\% (C) images: the red lines represent the GTV. CCTA, coronary CT angiography; r4DCT-50\%, phase $50 \%$ of respiratory four-dimensional CT; GTV, gross target volume.

Although the factors affecting the outcome of SBRT are unclear, accurate movement evaluation and tracking of the arrhythmia target volume are the focus of our case presentation, which will certainly have positive effects. As shown in the flowchart in Figure 7, real-time imageguided SBRT treatment in a patient with refractory VT was completed, and the results indicate that this method was safe and highly effective in the short-term.

Primarily, we believe that the precise integration of EAM with CT simulation to accurately locate the target volume and movement range of the VT substrate is critical to the success of SBRT. Therefore, it is necessary to rely on an experienced cardiologist to determine the origin of the VT based on EAM against the reference anatomy and to delineate it on CCTA images as the GTV, for which a respiratory movement range can be generated on r4DCT images. Several studies have reported the registration and fusion workflow of EAM and CT images, providing an objective reference for cardiologists $(5,6)$.

For immobilization, many centers use vacuum bags with 

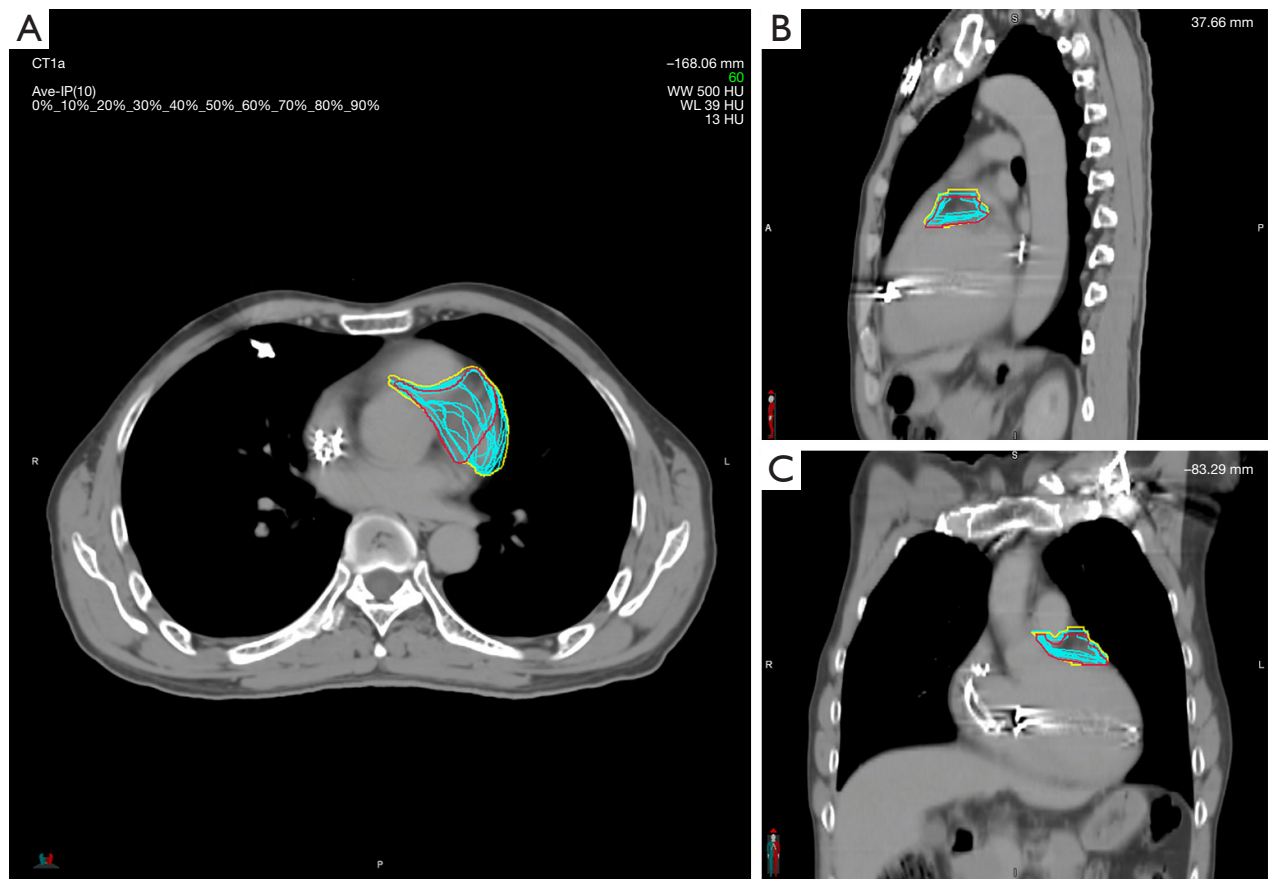

Figure 3 Motion evaluation of GTV from 10 phases on pCT: the red line represents the GTV obtained from r4DCT-50\%, the blue lines represent the GTV obtained from other phases, and the yellow line represents the ITV. GTV, gross target volume; pCT, planning CT; r4DCT-50\%, phase $50 \%$ of respiratory four-dimensional CT; ITV, internal target volume.
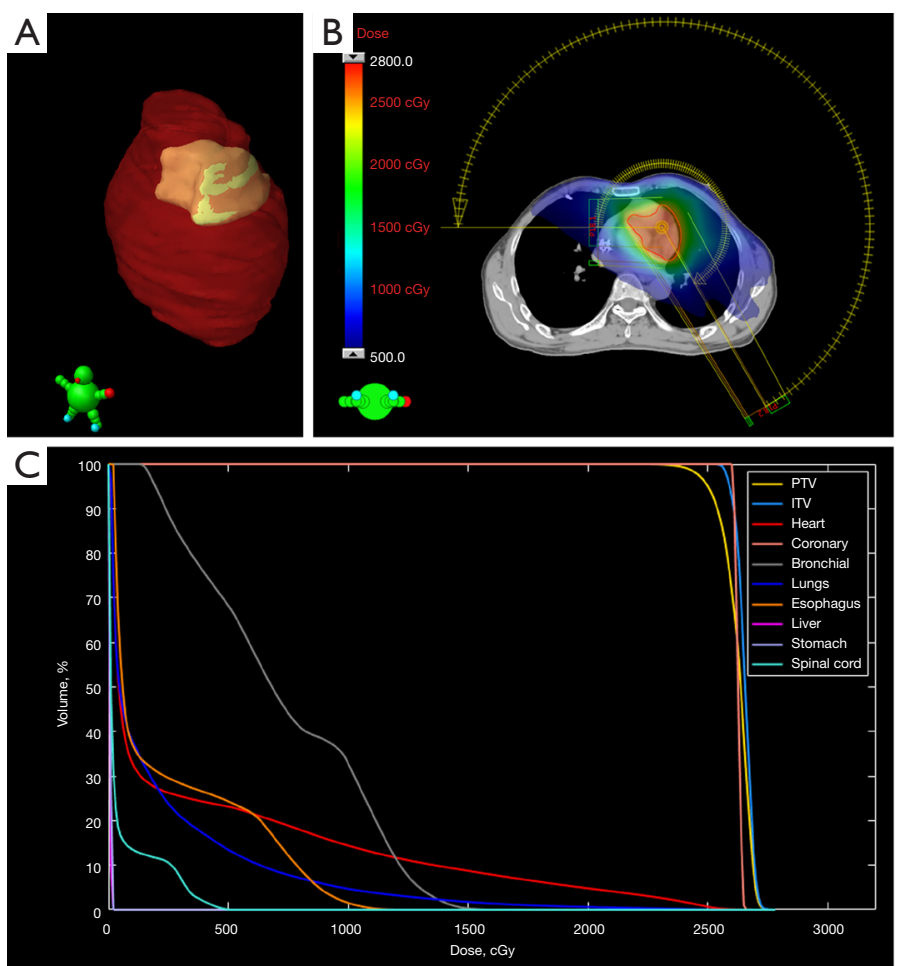

\begin{tabular}{|c|c|}
\hline $\begin{array}{c}\text { Dose } \\
\text { parameter }\end{array}$ & Value \\
\hline$D_{\min }(G y)$ & 22.30 \\
\hline$D_{\max }(G y)$ & 27.89 \\
\hline$D_{\text {mean }}(G y)$ & 26.22 \\
\hline PDC (Gy) & 95.00 \\
\hline R50 & 3.71 \\
\hline $\mathrm{HI}$ & 0.0985 \\
\hline $\mathrm{Cl}$ & 1.02 \\
\hline GI & 3.62 \\
\hline \begin{tabular}{|l}
$D_{\min }-$ minimum do \\
$D_{\max }$-maximum d \\
$D_{\text {mean }}$-mean dose \\
prescription dose \\
volume of PTV cC \\
R50-ratio of the \\
isodose to the PT \\
index, (D2-D98)/L \\
$n \%$ volume of PT \\
index, ratio of the \\
25 Gy isodose to \\
Gl-gradient index \\
volume of $12.5 \mathrm{G}$ \\
isodose
\end{tabular} & $\begin{array}{l}\text { of PTV; } \\
\text { of PTV; } \\
\text { PTV; PDC- } \\
\text { verage, } \\
\text { ed by } 25 \text { Gy; } \\
\text { me of } 12.5 \text { Gy } \\
\text { HI-homogeneity } \\
\text { (Dn is dose of } \\
\text { Cl-conformal } \\
\text { lume of the } \\
\text { PTV volume; } \\
\text { tio of the } \\
\text { odose to } 25 \text { Gy }\end{array}$ \\
\hline
\end{tabular}

Figure 4 PTV position and dose evaluation of the treatment plan: PTV position (A), dose distribution (B), dose volume histogram (C), dose parameters (D). PTV, planning target volume. 

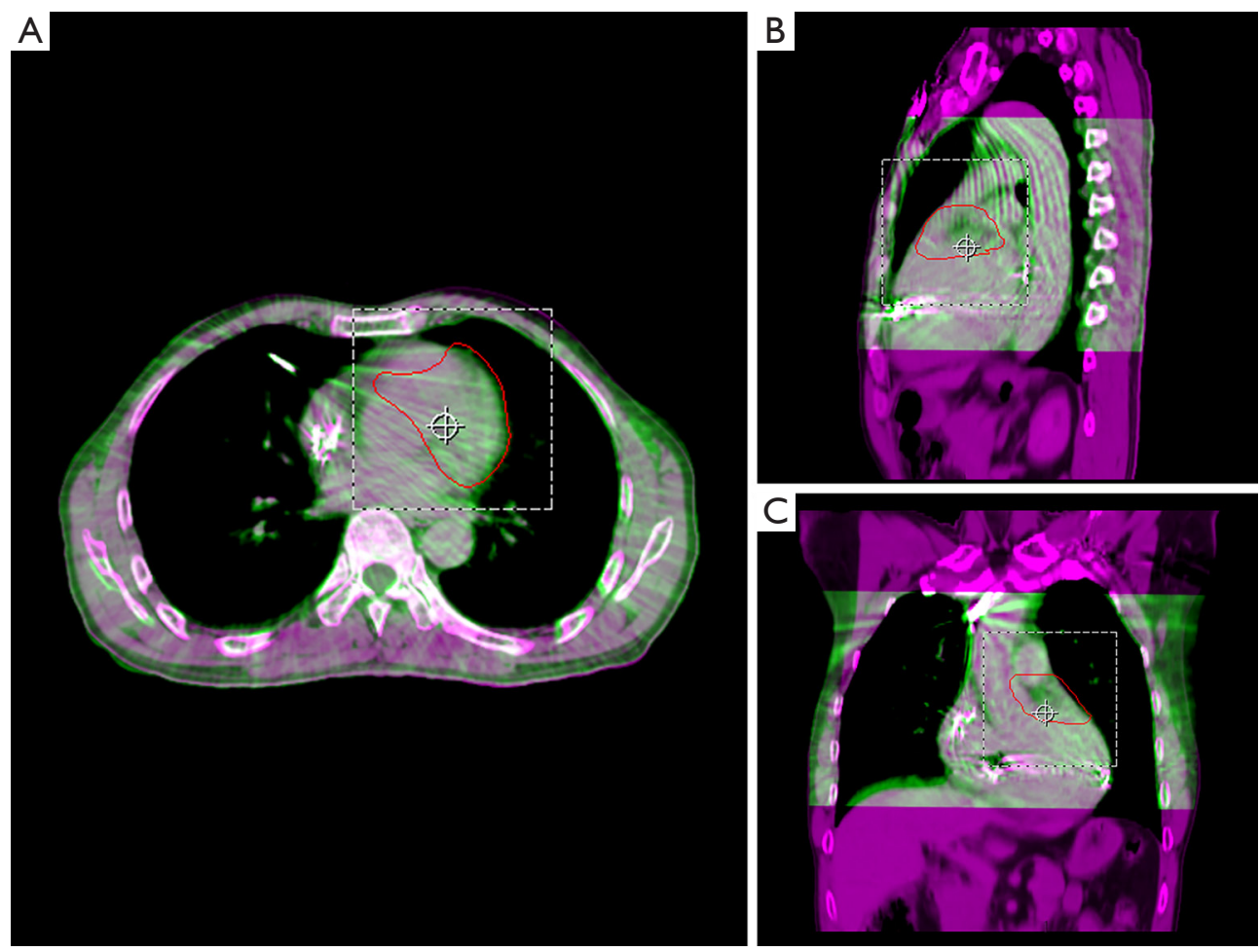

Figure 5 Registration of CBCT and pCT images: the green image pertains to CBCT, the red image pertains to pCT, and the red line represents PTV. CBCT, cone beam CT; pCT, planning CT; PTV, planning target volume.
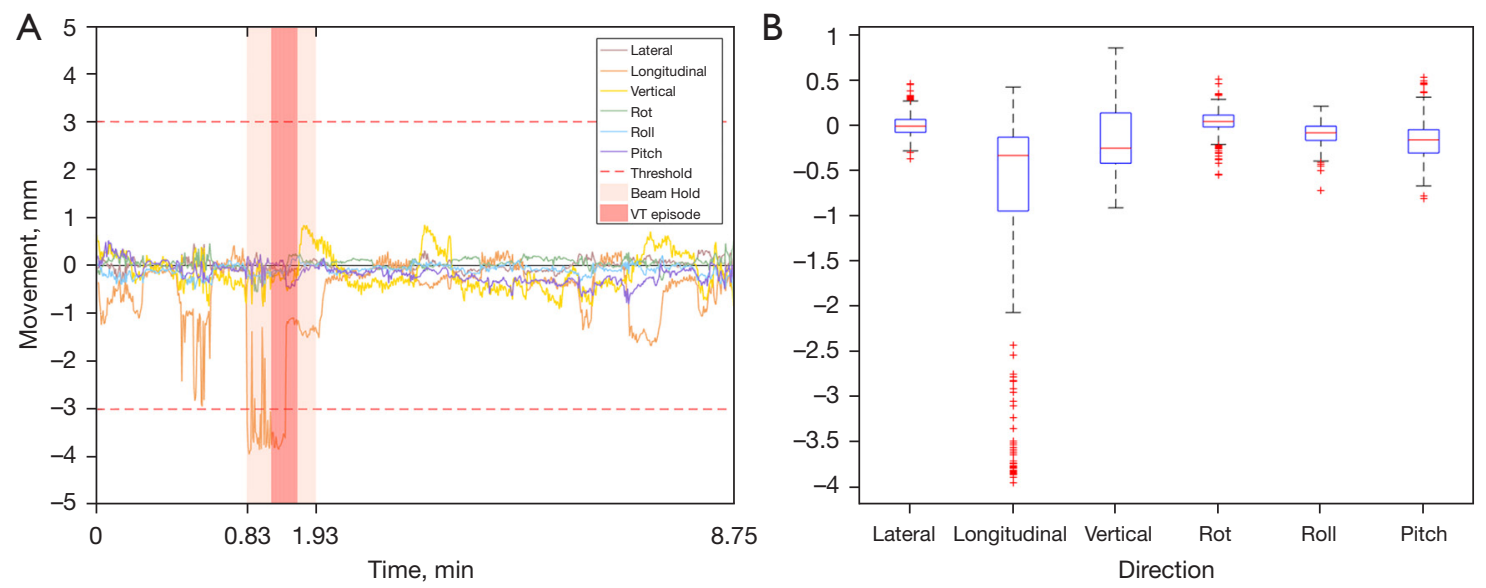

Figure 6 Results of body surface tracking during treatment (A) and statistical analysis (B).

negative pressure membranes or abdominal-pressure plates, which represent a significant challenge to patients. Another option might be to use a vacuum bag alone and expose the patient's body surface for OSI to enable the monitoring of surface movement in real time (7). To account for the movement of the GTV, which is affected by heart rhythm and respiratory motion, we collected CCTA and r4DCT sequences and used them to reconstruct pCT and imageguided reference images. Furthermore, it is convenient to perform registration and fusion for CT images obtained within the same coordinate system. Gating techniques are applied to ensure the accuracy of beam delivery; to achieve 


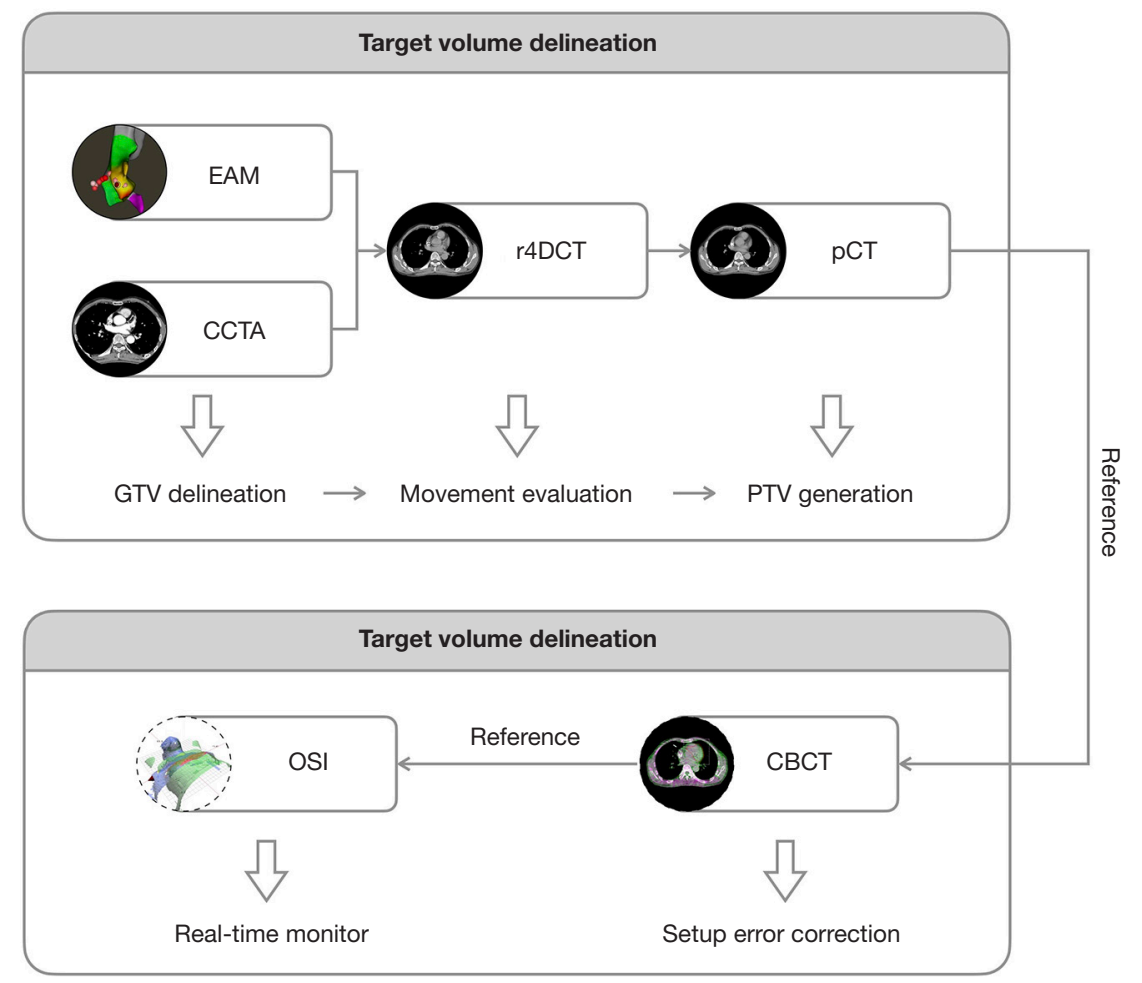

Figure 7 Overall workflow of image-guided stereotactic arrhythmia radiotherapy. EAM, electroanatomic mapping; CCTA, coronary CT angiography; r4DCT, respiratory four-dimensional CT; pCT, planning CT; GTV, gross target volume; PTV, planning target volume; OSI, optical surface imaging; CBCT, cone beam CT.

this goal in our study, a ROI on the patient's body surface was selected as a surrogate for the PTV, and real-time OSI was performed to interlock beam delivery with the surrogate movements.

There are several limitations to the use of SBRT in VT treatment. Although several study results have shown significant improvement in cardiac function with no VT recurrence in the short-term, long-term follow-up results and research on the mechanism by which high-energy rays eliminate the arrhythmia are lacking. Furthermore, despite individual cases having reported PTV maximum doses exceeding 30 Gy and different fractional patterns such as $24 \mathrm{~Gy} / 3$ fractions, the most widely used prescription is a single-fraction of $25 \mathrm{~Gy}$ (8-11). In terms of protecting the OARs from radiation, SBRT used for arrhythmia treatment is implemented as single-fraction high-dose radiotherapy for which there is no uniform reference regarding the relative biological effectiveness. Long-term follow-up studies have reported a strong correlation between coronary artery damage and death due to heart disease in patients receiving thoracic radiotherapy; however, excessive protection of the coronary arteries can lead to reduced dose coverage. In our case, the PTV partially overlapped with a coronary artery. To avoid insufficient PTV radiation dose, we ensured the uniformity of the dose distribution and prevented the hotspot from the coronary artery range, and no coronary artery damage occurred during short-term follow-up. Therefore, clinicians should make decisions based on the patient's condition, especially whether to deliver a sufficient dose to the target volume or to strictly constraint the OAR dose. Additionally, the relative biological effectiveness and OAR dose limitations will need to be verified in future clinical trials.

Despite the existence of outstanding questions regarding SBRT in arrhythmia treatment, our encouraging initialstage results indicate that the proposed treatment is quite promising. However, this clinically significant outcome should be further assessed in long-term follow-up studies for effectiveness and complications. The next step will be to conduct a multicenter clinical trial to refine protocols for patient selection, treatment planning, and the delivery of SBRT. The exact mechanism of radiotherapy in the 
treatment of arrhythmia also needs to be studied, which will require collaboration among cardiologists, oncologists, and physicists and will hopefully promote the development of new approaches.

\section{Acknowledgments}

Funding: This work was supported by the National Natural Science Foundation of China (No. 81972848), Cancer Precision Radiotherapy Spark Program of China International Medical Foundation (No. 2019-N-11-04), and the Sichuan Science and Technology Program (No. 2021YFS0143).

\section{Footnote}

Conflicts of Interest: All authors have completed the ICMJE uniform disclosure form (available at https://qims. amegroups.com/article/view/10.21037/qims-21-1025/coif). The authors have no conflicts of interest to declare.

Ethical Statement: The authors are accountable for all aspects of the work in ensuring that questions related to the accuracy or integrity of any part of the work are appropriately investigated and resolved. All procedures performed in this study were in accordance with the ethical standards of the institutional and/or national research committee(s) and with the Helsinki Declaration (as revised in 2013). Written informed consent was obtained from the patient for publication of this case report and accompanying images. A copy of the written consent is available for review by the editorial office of this journal.

Open Access Statement: This is an Open Access article distributed in accordance with the Creative Commons Attribution-NonCommercial-NoDerivs 4.0 International License (CC BY-NC-ND 4.0), which permits the noncommercial replication and distribution of the article with the strict proviso that no changes or edits are made and the original work is properly cited (including links to both the formal publication through the relevant DOI and the license). See: https://creativecommons.org/licenses/by-nc-nd/4.0/.

\section{References}

1. Peichl P, Sramko M, Cvek J, Kautzner J. A case report of successful elimination of recurrent ventricular tachycardia by repeated stereotactic radiotherapy: the importance of accurate target volume delineation. Eur Heart J Case Rep 2021;5:ytaa516.

2. Miszczyk M, Jadczyk T, Gołba K, Wojakowski W, Wita K, Bednarek J, Blamek S. Clinical Evidence behind Stereotactic Radiotherapy for the Treatment of Ventricular Tachycardia (STAR)-A Comprehensive Review. J Clin Med 2021;10:1238.

3. Knutson NC, Samson PP, Hugo GD, Goddu SM, Reynoso FJ, Kavanaugh JA, Mutic S, Moore K, Hilliard J, Cuculich PS, Robinson CG. Radiation Therapy Workflow and Dosimetric Analysis from a Phase 1/2 Trial of Noninvasive Cardiac Radioablation for Ventricular Tachycardia. Int J Radiat Oncol Biol Phys 2019;104:1114-23.

4. Brownstein J, Afzal M, Okabe T, Harfi TT, Tong MS, Thomas E, Hugo G, Cuculich P, Robinson C, Williams TM. Method and Atlas to Enable Targeting for Cardiac Radioablation Employing the American Heart Association Segmented Model. Int J Radiat Oncol Biol Phys 2021;111:178-85.

5. Brett CL, Cook JA, Aboud AA, Karim R, Shinohara ET, Stevenson WG. Novel Workflow for Conversion of Catheter-Based Electroanatomic Mapping to DICOM Imaging for Noninvasive Radioablation of Ventricular Tachycardia. Pract Radiat Oncol 2021;11:84-8.

6. Hohmann S, Henkenberens C, Zormpas C, Christiansen H, Bauersachs J, Duncker D, Veltmann C. A novel opensource software-based high-precision workflow for target definition in cardiac radioablation. J Cardiovasc Electrophysiol 2020;31:2689-95.

7. Narducci ML, Cellini F, Placidi L, Boldrini L, Perna F, Bencardino G, Pinnacchio G, Bertolini R, Cannelli G, Frascino V, Tagliaferri L, Chiesa S, Mattiucci GC, Balducci M, Gambacorta MA, Rossi M, Indovina L, Pelargonio G, Valentini V, Crea F. Case Report: A Case Report of Stereotactic Ventricular Arrhythmia Radioablation (STAR) on Large Cardiac Target Volume by Highly Personalized Inter- and Intra-fractional Image Guidance. Front Cardiovasc Med 2020;7:565471.

8. Loo BW Jr, Soltys SG, Wang L, Lo A, Fahimian BP, Iagaru A, Norton L, Shan X, Gardner E, Fogarty T, Maguire P, Al-Ahmad A, Zei P. Stereotactic ablative radiotherapy for the treatment of refractory cardiac ventricular arrhythmia. Circ Arrhythm Electrophysiol 2015;8:748-50.

9. Scholz EP, Seidensaal K, Naumann P, André F, Katus HA, Debus J. Risen from the dead: Cardiac stereotactic ablative radiotherapy as last rescue in a patient with refractory ventricular fibrillation storm. HeartRhythm Case Rep 
2019;5:329-32.

10. Qian PC, Azpiri JR, Assad J, Gonzales Aceves EN, Cardona Ibarra CE, de la Pena C, Hinojosa M, Wong D, Fogarty T, Maguire P, Jack A, Gardner EA, Zei PC. Noninvasive stereotactic radioablation for the treatment of atrial fibrillation: First-in-man experience. J Arrhythm
2019;36:67-74.

11. Park JS, Choi Y. Stereotactic Cardiac Radiation to Control Ventricular Tachycardia and Fibrillation Storm in a Patient with Apical Hypertrophic Cardiomyopathy at Burnout Stage: Case Report. J Korean Med Sci 2020;35:e200.

Cite this article as: Li J, Chen Q, Li G, Li Y, Zhang Y, Li C, Bai L, Zhong R, He Y, Xu F, Yang Q, Bai S. Stereotactic arrhythmia radiotherapy: a case study of real-time image-guided noninvasive treatment for ventricular tachycardia. Quant Imaging Med Surg 2022;12(4):2607-2615. doi: 10.21037/qims-211025 